\title{
Low Data Rate Ultra Wideband ECG Monitoring System
}

\author{
Ho Chee Keong and Mehmet R. Yuce, Member, IEEE
}

\begin{abstract}
This paper presents a successfully implemented wireless electrocardiograph monitoring using low data rate ultra wideband (UWB) transmission. Low data rate ultra wideband is currently under consideration for the newly formed wireless body area network (WBAN) group (IEEE802.15.6) to develop a standard for wireless vital sign monitoring. Maximizing the transmission power of the transmitter and reducing the stringent requirements and complexity of the receiver have always been the key considerations for an UWB transceiver. Multiple pulses per bit has been sent in our low data rate UWB prototype system to increase the transmitter power, to reduce the complexity of the receiver and to ease the requirement on the receiver's analog to digital converter. Non-coherent technique has been used for the demodulation of UWB signals at the receiver that reduces the receiver complexity further.
\end{abstract}

\section{INTRODUCTION}

$\mathrm{T}$ HE emergence of low power and robust wireless biotelemetry devices has increased patient mobility and the efficiency of medical staff. Monitoring vital signs for patients that require long term care can now be performed remotely in the patient's comfort, allowing medical staff to monitor several patients simultaneously.

Numerous wireless communication technologies and protocols are proposed for biotelemetry systems. In November 2007, a working group for the Body Area Network (BAN) known as IEEE802.15.6 was formed to develop a communication standard optimized for low power devices operating near or in a human body [1]. IEEE802.15.4a, a low data rate ultra wideband (UWB) based standard, which was approved in March 2007 is one of the potential candidates for the body area network [2]. Its transmission power is lower than other wireless personnel network (WPAN) standards and offers a data rate throughput of $850 \mathrm{kbps}$, which is suitable for monitoring of multiple continuous electrocardiogram (ECG) and electroencephalogram (EEG) signals [3].

This paper presents a single channel real-time wireless electrocardiograph monitoring system, which was implemented using low data rate ultra wideband impulse radio method, aligned with the direction of IEEE802.15.6. The system is designed to operate with a center frequency of $4 \mathrm{GHz}$ and a bandwidth of $1 \mathrm{GHz}$. The number of pulses per bit the transmitter send depends on the selection of clock rate. This feature increases the power level at the transmitted

This work was supported in part by the Australian Research Council (ARC) under Discovery Projects Grant.

Ho Chee Keong and M. R. Yuce are with the School of Electrical Engineering and Computer Science, University of Newcastle, Callaghan, NSW 2308, Australia (e-mail: mehmet.yuce@ newcastle.edu.au ).

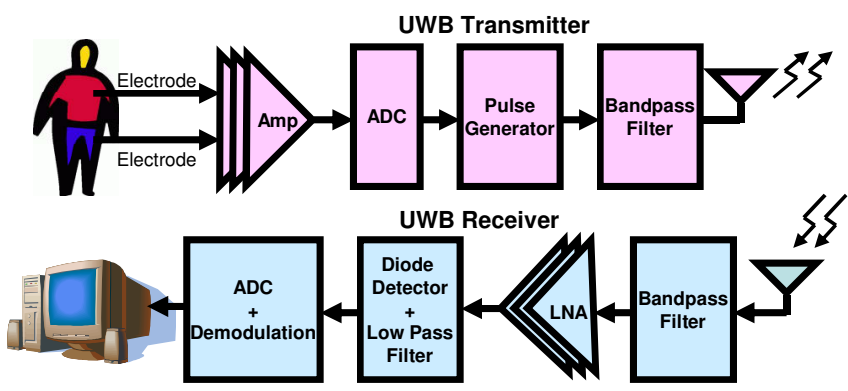

Figure 1: ECG transceiver block diagram

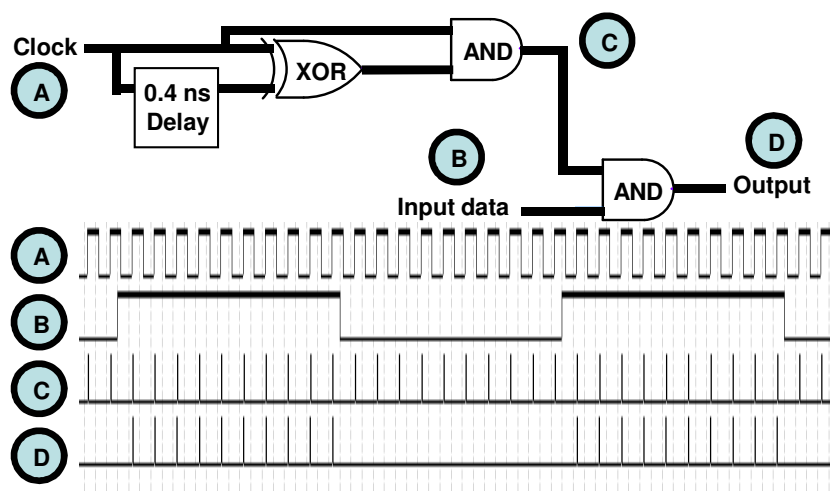

Figure 2: Pulse generation circuit and its time diagram

band at $4 \mathrm{GHz}$. A non-coherent energy detector receiver is used to demodulate the received signal. There are several advantages in using a non-coherent compared to a coherent receiver, especially in low data rate applications. It removes the need for a high speed analog to digital converter (ADC) and precise synchronization. The demodulated data is transmitted to the computer (PC) using the Universal Asynchronous Receiver/Transmitter (UART) format with the bit rate of $9600 \mathrm{bps}$. The DC 50/60 Hz noise is removed using a digital notch filter implemented in the PC. A block diagram for the implemented system is shown in Figure 1.

\section{TRANSMITTER}

The front-end of the ECG transmitter consists of an instrumental amplifier (INA321) [4] and an active low pass filter (AD712). The gain of INA321 is set at 5 and the active low pass filter has a cutoff frequency of $100 \mathrm{~Hz}$ and a gain of 200. A gain of $60 \mathrm{~dB}$ is produced by these two stages. INA321 takes in a pair of differential inputs and produces an amplified, level shifted single ended output. The virtual ground is set at $1.25 \mathrm{~V}$. The output of INA321 is fed into an active low pass filter, which conditions the signal before sending to the ADC. The ADC samples at $237 \mathrm{~Hz}$ and 


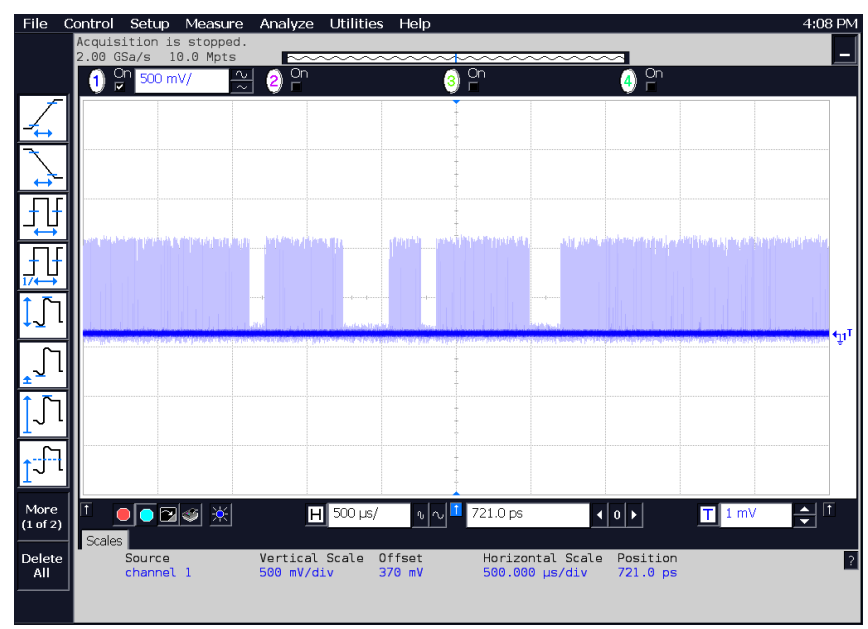

Figure 3: UWB output signal with a clock input of $50 \mathrm{MHz}$

produces a parallel bit stream at the output that is converted to a serial bit stream based on the UART format. The UWB pulses are generated using the circuit given in Figure 2. The circuit is implemented digitally using a field programmable gate array (FPGA). The input data coming from the ADC output is arranged to be $9600 \mathrm{bps}$ similar to the UART data rate. The width of the UWB pulse signal is selected to be 0.4 ns, which is controlled by adjusting the delay unit. A $0.4 \mathrm{~ns}$ UWB signal is generated at the positive edge of each clock pulse (C). The output signal (D) is generated simply multiplying (C) with the data signal (B) using AND gate. The number of pulses per bit at the output is determined by the clock rate. The modulated output signal (D) using a 50 $\mathrm{MHz}$ clock is illustrated in Figure 3.

To obtain maximum performance in ultra wideband communications, the spectrum of the transmitted signal should be kept as close to the regulated spectrum mask [5] as possible. The strength of the high frequency components largely depends on the rate of rise and fall time of the UWB pulse. A step recovery diode can be used to sharpen the pulse or an amplifier can be used to boost the high spectral components. Both methods consume a fair amount of power. Alternatively, sending multiple pulses per bit is an option for low data rate medical monitoring applications.

As illustrated in Figure 4 with the spectrum plot of a 0.4 ns UWB pulse when clock rate of $500 \mathrm{kHz}, 50 \mathrm{MHz}$ and 500 $\mathrm{MHz}$ is used, the high frequency component increases when multiple pulses per bit are sent. The method not only improves the transmission range, it also eases the demodulation process and ensures high data reliability, which is an important factor for medical devices.

The input data in this prototype is based on the UART format. Each 10 bits data output from the ADC is transmitted using two bytes, each byte come with one start bit at the start of the byte and one stop bit at the end, which forms a periodic sequence. The digital UWB pulses can be represented by (1), which is the Fourier series expansion of a rectangular pulse train [6]. The UWB pulses are passed through a wideband bandpass filter (BPF)

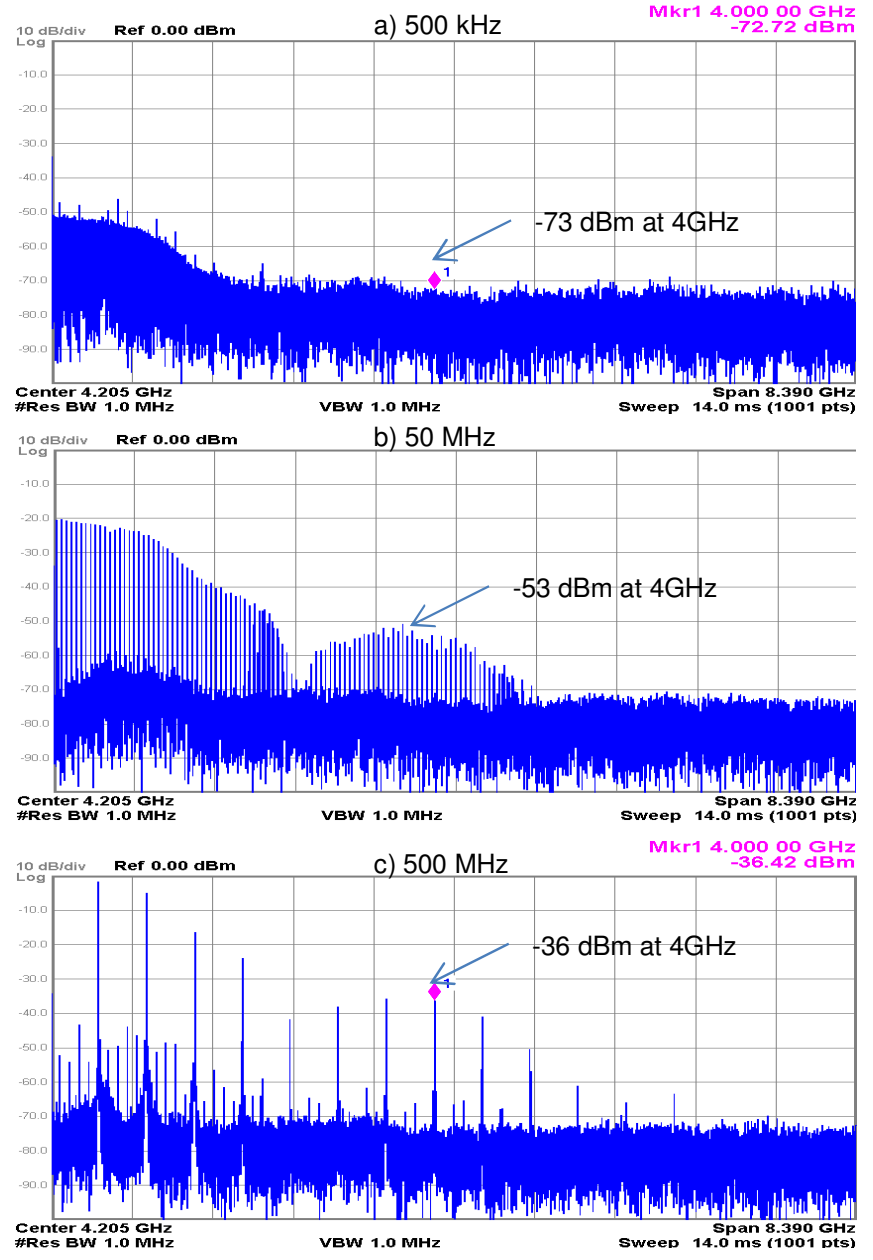

Figure 4: Spectrum plot for different PRF

centered at $4 \mathrm{GHz}$ to ensure the UWB spectrum mask requirements are met. Since the UWB signal will be band limited after passing through the BPF, it can therefore be described by (2).

$$
\begin{gathered}
x(t)=\frac{A \tau}{T_{b}}+\frac{2 A \tau}{T_{b}} \sum_{k=1}^{\infty} \frac{\sin \left(\pi k \tau / T_{b}\right)}{\pi k \tau / T_{b}} \cos (k \omega t) \\
x(t)=\frac{2 A \tau}{T_{b}} \sum_{k=n 1}^{n 2} \frac{\sin \left(\pi k \tau / T_{b}\right)}{\pi k \tau / T_{b}} \cos (k \omega t)
\end{gathered}
$$

where $A$ is the pulse amplitude, $T_{b}$ is the period of the clock (50 $\mathrm{MHz}$ in this paper), $\tau$ is the pulse width, $\omega=2 \pi / T_{b}$, $n 1=\omega / \omega_{1}, n 2=\omega / \omega_{2}, \omega_{1}$ and $\omega_{2}$ are the lower and upper cutoff frequency of the bandpass signal.

Selecting the appropriate pulse width and pulse repetitive frequency (PRF) is the key to an optimized system. PRF is determined by the clock rate. From (2), it can be observed that for a given bandwidth, the number of the spectrum lines increases as the PRF reduces. High PRF will result in less spectral line with higher amplitude, while a low PRF will have more spectral lines of lower amplitude. Therefore, a low data rate ultra wideband system which is able to use a lower PRF can transmit more power than high data rate 


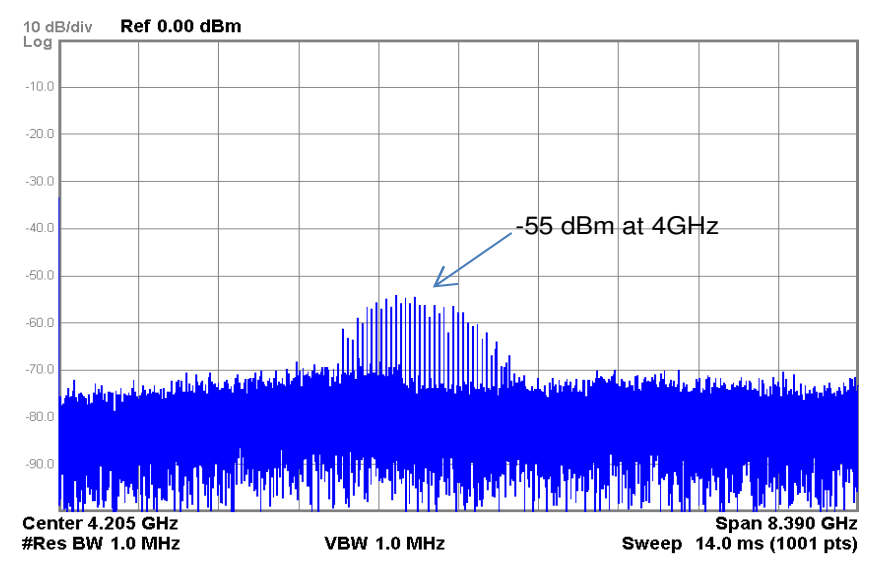

Figure 5: Spectrum plot of transmitted signal

system while not exceeding the $-41 \mathrm{dBm}$ requirement. As the low data rate signal has more spectral lines, it will have a higher total energy after the amplification on the receiver end, which works well with a non coherent energy detector. In our prototype, PRF of $50 \mathrm{MHz}$ works well with our system. As illustrated in Figure 4, selecting a PRF that is too low will result in insufficient transmission power.

Pulse width is another important consideration, null occurs every integer multiple of $1 / \tau$. The optimized pulse width for the system is selected to be $0.4 \mathrm{~ns}$, this ensures that null does not occurs in the band of interest. Figure 5 shows the spectrum plot of the transmitted signal, which is the signal after passing through the bandpass filter.

\section{RECEIVER}

At the receiver, the received signal is passed through a 1 $\mathrm{GHz}$ bandpass filter centered at $4 \mathrm{GHz}$ to remove the interfering signals from other narrowband devices. A four stage low noise amplifier (LNA) circuit is used to amplify the received signal by $50 \mathrm{~dB}$. All the measurements are taken with the receiver placed $30 \mathrm{~cm}$ away from the transmitter. Figure 6 shows the spectrum plot of the received signal with a $50 \mathrm{MHz}$ clock input after the amplification, there is a loss of $35 \mathrm{~dB}$. Based on the famous Friis formula shown in (3), the calculated free space path loss is $34 \mathrm{~dB}$ for a distance of $30 \mathrm{~cm}$, which is close to our measured losses.

The heart of the receiver circuit is the diode detector. It translates the high frequency components to their equivalent low frequency counterparts and forms an envelope, which greatly eases the stringent requirements on the ADC. Operation of the diode detector can be described by EbersMoll (4) [7]. When a low input signals below $-20 \mathrm{dBm}$ is applied to the diode, it can be represented by (5), which is the first two terms of the Taylor series expansion of (4). The first term is the square law output from the diode, which are the translated low frequency components that we are interested in. (6) shows the diode response to the band limited input given in (2). From (6), the square law detector responds better to low data rate UWB signal where there is less numbers of zero spectral components in the band. Zeros

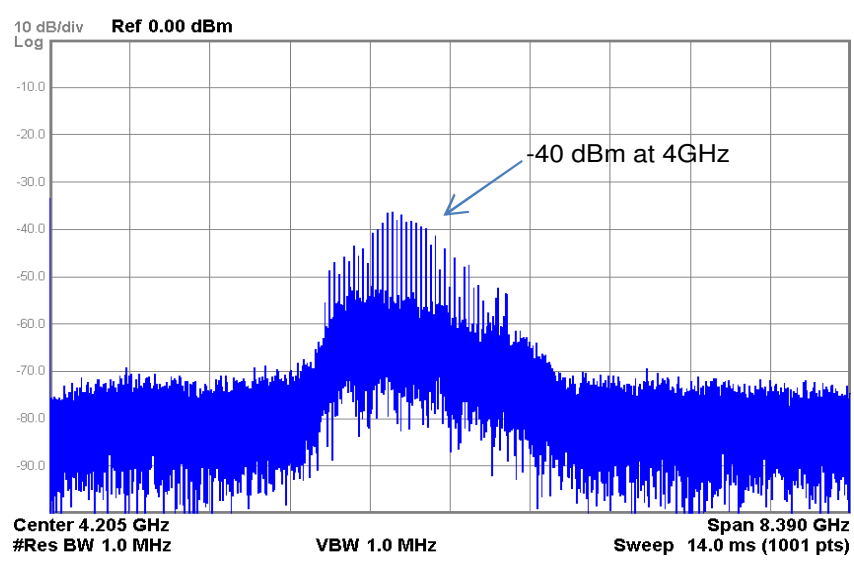

Figure 6: Spectrum plot of received signal

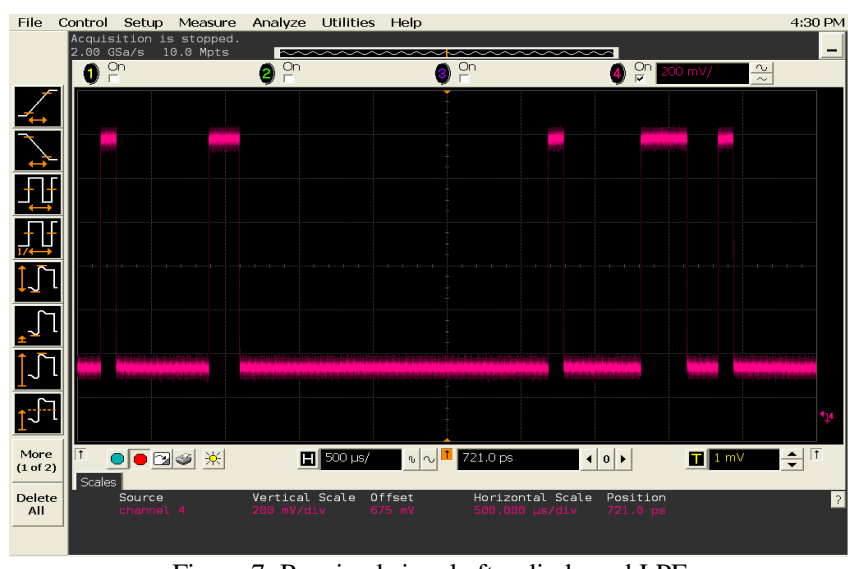

Figure 7: Received signal after diode and LPF

$$
\text { Path Loss }=20 \log _{10}(4 \pi d / \lambda)
$$

occurs every $k \tau=T_{b}$ interval.

The received signal after passing through the diode detector and the low pass filter is shown in Figure 7. The use of multiple pulses eases the formation of an envelope for the baseband data, which greatly improved the performance and relaxed the ADC requirement. A simple comparator or slow speed 1 bit ADC can be used to digitize the received data, which greatly reduces the power consumption and complexity of an ultra wideband receiver.

$$
\begin{gathered}
I=I_{s}\left(e^{\alpha V}-1\right), \quad \alpha=\frac{q}{n k T} \\
I=I_{s}\left(\alpha V+\frac{(\alpha V)^{2}}{2 !}\right) \\
I=I_{s} \sum_{k=n 1}^{n 2}\left(\begin{array}{l}
\left.\left(\frac{\alpha A}{\pi k}\right)^{2}\left(1-\cos \left(\frac{2 \pi k \tau}{T_{b}}\right)\right)+\alpha\left(\frac{2 A \tau}{T_{b}}\right) \frac{\sin \left(\pi k \tau / T_{b}\right)}{\pi k \tau / T_{b}} \cos (k \omega t)\right) \\
+\left(\frac{\alpha A}{\pi k}\right)^{2}\left(1-\cos \left(\frac{2 \pi k \tau}{T_{b}}\right)\right) \cos (2 k \omega t)
\end{array}\right)
\end{gathered}
$$




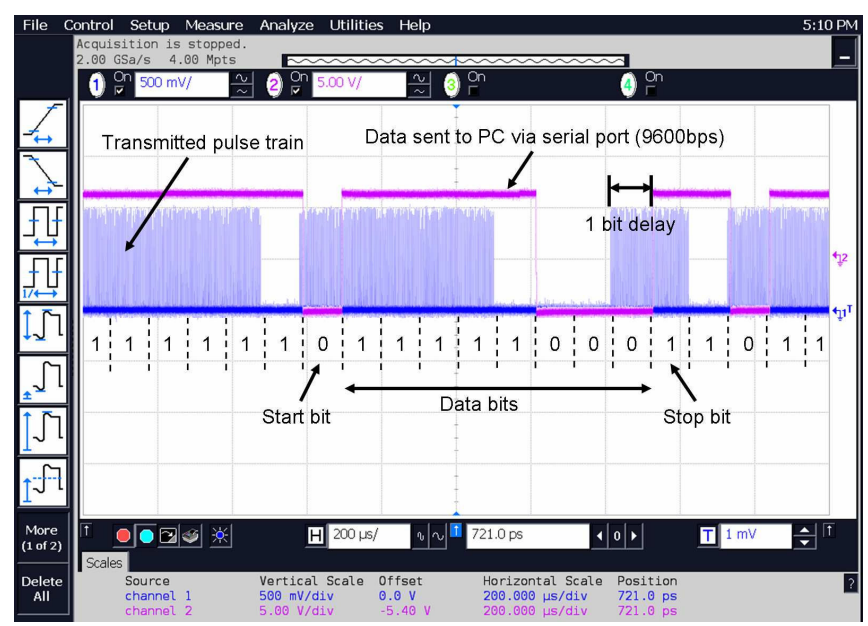

Figure 8: Demodulated data

The demodulation is carried out using an FPGA. The output format is a 9600 bps UART bit stream. The demodulator counts the number of '1's in the period of one bit, which is approximately $104 \mu \mathrm{s}$. The output will be a high if it exceeds certain numbers of '1's. A demodulated data together with the transmitted pulse train is shown in Figure 8. The demodulated signal is one bit period slower than the transmitted signal. This delay is due to the processing time required for counting the number of ' 1 's in the bit period.

The demodulated data is sent to the PC via RS232 serial port. The received data is checked for errors and the corrupted bytes are removed. The result is displayed using Matlab in Fig 9. The signal is corrupted by the $50 \mathrm{~Hz}$ noise as can been seen in the waveform obtained from the oscilloscope before transmitting (Fig 9(a)), after receiver and monitoring in Matlab in time (Fig 9(b)) and the frequency domain (c). The signal is passed through a $50 \mathrm{~Hz}$ digital notch filter designed using a Matlab program and the result in Figure 10. The $50 \mathrm{~Hz}$ noise is successfully removed and the ECG signal recovered. Removing the $50 \mathrm{~Hz}$ noise at the PC instead of the receiver helps to reduce the complexity and the programming power required at the receiver. The whole measurement has been carried out in our lab where there were other wireless standards (e.g WiFi) and equipments operating. The ECG signal has successfully been monitoring without any error.

\section{CONCLUSION}

A low data rate ultra wideband system for monitoring ECG signal has successfully been implemented. The low data rate nature of the UWB system is in the direction of IEEE802.15.6. Multiple UWB pulses are used per information bit at the transmitter that improves the data reliability and reduces the power consumption as well as the complexity of the receiver. It has also been shown that a low data rate ultra wideband system performs better than a high data rate ultra wide system when used with a non-coherent energy detector. A single channel ECG signal has
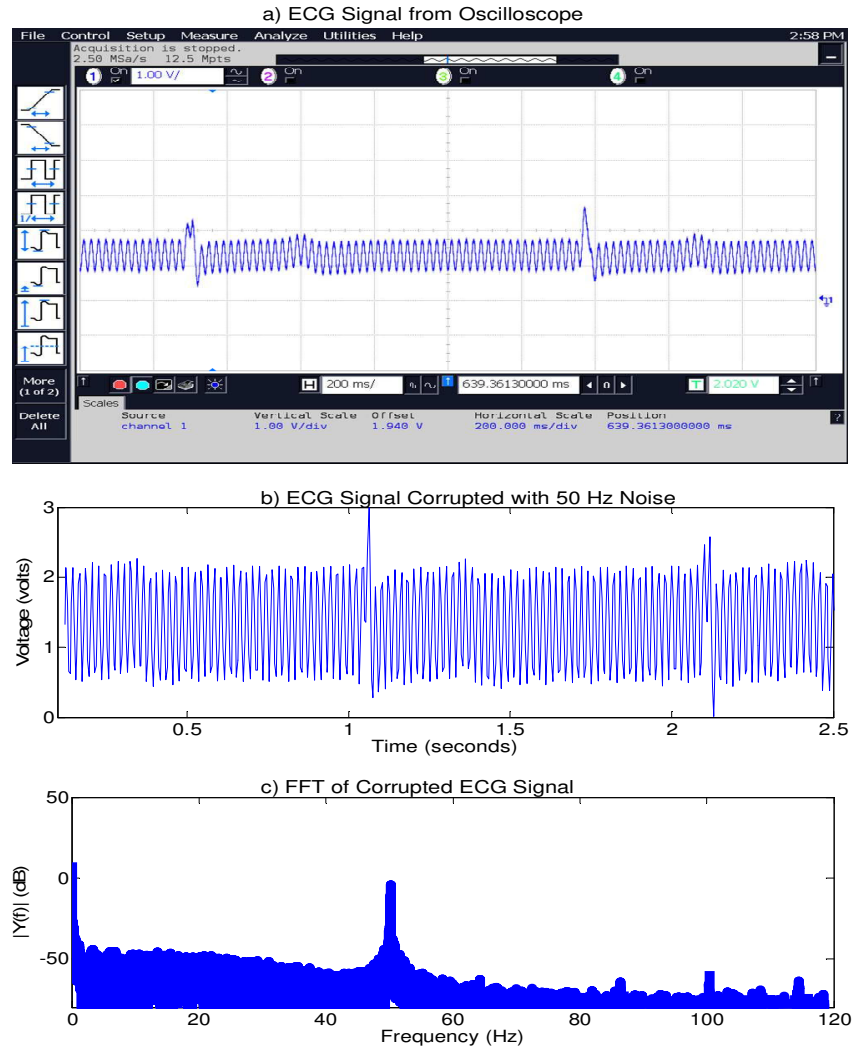

Figure 9: ECG waveform with $50 \mathrm{~Hz}$ noise

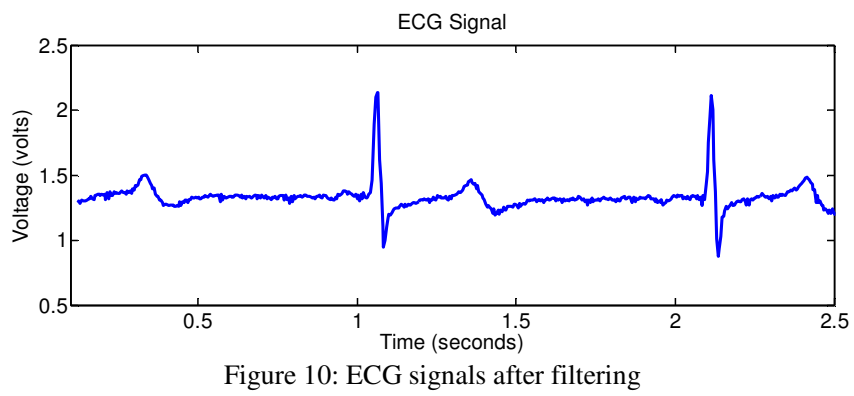

successfully been monitored in an environment where other wireless standards are also operating, without any interference effect. In the future, we will experiment with multi-channel ECG/EEG signals as well as incorporate other signals such as temperature and pulse rate.

\section{REFERENCES}

[1] http://www.iee802.org/15/pub/TG6.html, 2008.

[2] H. B. Li, K. Takizawa, B. Zhen and R. Kohno, "Body area network and its standardization at IEEE802.15.MBAN," Mobile and Wireless Communication Summit, pp. 1-5, 2007

[3] K. Takizawa, H. B. Li, K. Hamaguchi and R. Kohno, "Wireless vital sign monitoring using ultra wideband-based personal area networks ," Engineering in Medicine and Biology Society. EMBS. 29th Annual International Conference of the IEEE, pp. 1798-1801, 2007

[4] http://focus.ti.com/lit/ds/symlink/ina321.pdf

[5] http://hraunfoss.fcc.gov/edocs_public/attachmatch/FCC-02-48A1.pdf

[6] M. R. Yuce, W. Liu, M. S. Chae, and J. S. Kim, "A Wideband Telemetry Unit for Multi-Channel Neural Recording Systems," IEEE International Conference on Ultra-Wideband, 2007.

[7] R. Ludwig and P. Bretchko, RF circuit design: theory and applications. Prentice Hall,2000. 